\title{
Characterization and Genomic Analysis of SFPH2, a Novel T7virus Infecting Shigella
}

\author{
Chaojie Yang ${ }^{1 \dagger}$, Haiying Wang ${ }^{21}$, Hui Ma ${ }^{3 \dagger}$, Renlong Bao ${ }^{1}$, Hongbo Liu', Lang Yang ${ }^{1}$, \\ Beibei Liang ${ }^{1}$, Leili Jia ${ }^{1}$, Jing Xie ${ }^{1}$, Ying Xiang ${ }^{1}$, Nian Dong ${ }^{1}$, Shaofu Qiu ${ }^{1 *}$ and \\ Hongbin Song ${ }^{1 *}$
}

${ }^{1}$ Institute of Disease Control and Prevention of PLA, Beijing, China, ${ }^{2}$ Joint Service Academy, National Defence University of People's Liberation Army, Beijing, China, ${ }^{3}$ The 6th Medical Center of Chinese PLA General Hospital, Beijing, China

\section{OPEN ACCESS}

Edited by:

Robert Czajkowski,

University of Gdańsk, Poland

Reviewed by:

Elizabeth Martin Kutter,

The Evergreen State College,

United States

Olivia McAuliffe,

Teagasc, The Irish Agriculture and Food Development Authority,

Ireland

*Correspondence:

Shaofu Qiu

qiushf0613@hotmail.com

Hongbin Song

hongbinsong@263.net

†These authors have contributed equally to this work

Specialty section:

This article was submitted to

Virology,

a section of the journal

Frontiers in Microbiology

Received: 14 September 2018 Accepted: 22 November 2018

Published: 14 December 2018

Citation:

Yang $C$, Wang $H, M a H$, Bao $R$, Liu H, Yang L, Liang B, Jia L, Xie J, Xiang Y, Dong N, Qiu S and Song $H$ (2018) Characterization and Genomic Analysis of SFPH2, a Novel T7virus Infecting Shigella.

Front. Microbiol. 9:3027. doi: 10.3389/fmicb.2018.03027
Shigellosis, caused by Shigella, is a major global health concern, with nearly 164.7 million cases and over a million deaths occurring annually worldwide. Shigella flexneri is one of the most common subgroups of Shigella with a high incidence of multidrugresistance. The phage therapy approach is an effective method for controlling multidrugresistant bacteria. However, only a few Shigella phages have been described to date. In this study, a novel lytic bacteriophage SFPH2 was isolated from a sewage sample obtained from a hospital in Beijing, China, using a multidrug-resistant $S$. flexneri 2a strain (SF2) isolated from the fecal sample of a dysentery patient. SFPH2 is a member of the Podoviridae virus family with an icosahedral capsid and a short, non-contractile tail. It was found to be stable over a wide range of temperatures $\left(4-50^{\circ} \mathrm{C}\right)$ and $\mathrm{pH}$ values (pH 3-11). Moreover, SFPH2 could infect two other S. flexneri serotypes (serotypes 2 variant and $\mathrm{Y}$ ). High-throughput sequencing revealed that SFPH2 has a linear doublestranded DNA genome of 40,387 bp with 50 open reading frames. No tRNA genes were identified in the genome. Comparative analysis of the genome revealed that the SFPH2 belongs to the subfamily Autographivirinae and genus T7virus. The genome shows high similarity with other enterobacterial T7virus bacteriophages such as Citrobacter phage SH4 (95\% identity and 89\% coverage) and Cronobacter phage Dev2 (94\% identity and 92\% coverage). A comparison of the fiber proteins showed that minor differences in the amino acid residues might specify different protein binding regions and determine host species. In conclusion, this is the first report of a T7virus that can infect Shigella; SFPH2 has a functional stability under a wide range of temperatures and $\mathrm{pH}$ values, showing the potential to be widely applied to control Shigella-associated clinical infections and reduce the transmission rates of $S$. flexneri serotype $2 a$ and its variants in the environment.

Keywords: bacteriophage, Shigella flexneri, T7virus, genome analysis, tail fiber

\section{INTRODUCTION}

Shigellosis, an infectious diarrheal disease, is caused by the enteric pathogen Shigella. It is a major worldwide health burden and causes nearly 164.7 million cases and over a million deaths every year, most of them occurring in developing countries (Kotloff et al., 1999). Shigella flexneri, one of the four Shigella subgroups, is the most common cause of endemic shigellosis (Kotloff et al., 1999; 
von Seidlein et al., 2006). Based on its serology, S. flexneri can be further subdivided into at least 20 serotypes (serotypes 1a, 1b, 1c, 1d, 2a, 2b, 2 variant, 3a, 3b, 4a, 4av, 4b, 5a, 5b, X, Xv, Y, Yv, F6, and 7b) (Qiu et al., 2013; Sun et al., 2013). Serotypes 2 variant, Xv, and $\mathrm{Yv}$ are newly reported serotypes and are associated with epidemic-level disease (Ye et al., 2010; Sun et al., 2013; Qiu et al., 2015). Serotype 2 of S. flexneri (2a, 2b, and 2 variant) is the most prevalent among the 20 serotypes (Qiu et al., 2015) and has been recently reported to show a high frequency of multidrug-resistance (Zhang et al., 2011; Cui et al., 2015), which undoubtedly narrows the choice of effective antimicrobials. In February 2017, the World Health Organization designated Shigella as the priority target for the development of new antimicrobials (WHO, 2017). Therefore, the development of novel treatments or strategies has become an absolute necessity to control Shigella infections.

Phages are viruses that infect bacteria and are estimated to be the most abundant organisms on earth (Chibani-Chennoufi et al., 2004). Phage therapy has proven to be an effective method to control bacterial infection (Sulakvelidze et al., 2001) and it has been successfully applied for the treatment of Shigella dysenteriae infections in children (d'Herelle, 1931). However, relatively few studies aimed at isolating or characterizing Shigella phages have been performed. Only $\sim 42$ genome sequences of Shigella phages have been deposited with the NCBI nucleotide database and detailed studies of these phages are limited. In contrast, over 1,000 Escherichia and Salmonella phage sequences are readily available in the database. Phage host range is a significant feature of phage therapy and generally indicates the types (strains or species) of bacteria that the phage is able to infect (Hyman and Abedon, 2010). The tail fiber proteins of phages are thought to determine their host range and play an important role during the host recognition process (Garcia-Doval and van Raaij, 2013).

In this study, we report a novel Shigella lytic bacteriophage, SFPH2, which was isolated from a sewage sample using a multidrug-resistant $S$. flexneri 2 a strain. We examined its biological properties, determined its genomic sequence, and compared its genome and tail fiber protein sequences with other known phage sequences. To the best of our knowledge, this is the first report of a T7virus able to infect Shigella. Our research will contribute to the understanding of Shigella phages and the effective control of Shigellosis caused by Shigella.

\section{MATERIALS AND METHODS}

\section{Bacterial Strains and Serotyping}

The S. flexneri strains used in this study were isolated from the fecal samples of patients with diarrhea or dysentery. The fecal samples were screened for Shigella species as follows: samples were streaked onto Salmonella-Shigella (SS) agar and then incubated overnight at $37^{\circ} \mathrm{C}$. Suspected Shigella colonies were picked and streaked directly onto SS agar and incubated overnight at $37^{\circ} \mathrm{C}$. The resulting colonies were sub-cultured on Luria-Bertani (LB) agar plates and grown in a $37^{\circ} \mathrm{C}$ incubator. API 20E test strips (bioMérieux Vitek, Marcy-L'Etoile, France) were used for the identification of the Shigella strains following the manufacturer's recommendations. The specific serotypes of the strains were identified using monovalent antisera (Denka Seiken, Tokyo, Japan).

Informed and written consents were obtained from the patients for sample collection and usage, as well as for the publication of obtained results in this study. All experiments were approved and authorized by the Ethics Committees of the Institute of Disease Prevention and Control, People's Liberation Army, China.

\section{Phage Isolation and Purification}

Bacteriophage SFPH2 was isolated from a sewage sample obtained from the 307 Hospital of PLA in Beijing, China. The bacteriophage was purified, using the agar double-layer method (Kropinski et al., 2009), from a multidrug-resistant S. flexneri 2a strain (SF2) isolated from a fecal sample of a 20-month-old male child diagnosed with diarrhea.

Sodium chloride $(1 \mathrm{~mol} / \mathrm{L})$ and polyethylene glycol (PEG) 8000 (at a final concentration of $10 \%$ ) were added to the sewage samples and incubated at $4^{\circ} \mathrm{C}$ for $24 \mathrm{~h}$. Following centrifugation $\left(8,000 \times g\right.$ for $10 \mathrm{~min}$ at $\left.4^{\circ} \mathrm{C}\right)$, the supernatant was filtered using a $0.22-\mu \mathrm{m}$ membrane filter. Phages were pelleted by centrifugation at $12,000 \times g$ for $1 \mathrm{~h}$ at $4^{\circ} \mathrm{C}$ and resuspended in sterilized sodium chloride-magnesium sulfate (SM) buffer $(100 \mathrm{mmol} / \mathrm{L} \mathrm{NaCl}, 8$ $\mathrm{mmol} / \mathrm{L} \mathrm{MgSO}_{4}, 2 \%$ gelatin, and $50 \mathrm{mmol} / \mathrm{L}$ Tris- $\mathrm{HCl}$ [pH 7.5]). Spot tests were performed by spotting $0.05 \mathrm{~mL}$ of the phage solution onto bacterial lawns on a Luria-Bertani agar plate. Clear zone formations were assessed after overnight incubation.

To purify the phages, the double-layer agar plate method was used to collect single-plaque isolations, which were harvested and suspended in $0.5 \mathrm{~mL}$ of SM buffer. These steps were carried out at least five times in order to purify the phage. To concentrate the phage solution, $100 \mu \mathrm{L}$ of the purified phage solution was added to $5 \mathrm{~mL}$ of host bacterial culture fluid (LB medium, $\mathrm{OD}_{600}=0.5$ ) and incubated at $37^{\circ} \mathrm{C}$ for $12 \mathrm{~h}$. Culture samples were centrifuged at $12,000 \times g$ at $4^{\circ} \mathrm{C}$ for $10 \mathrm{~min}$ and filtered to remove cell debris. PEG 8000 (at a final concentration of 10\%) was added to the phage solution and the mixture was centrifuged at $12,000 \times g$ at $4^{\circ} \mathrm{C}$ for $10 \mathrm{~min}$. Subsequently, the solution was incubated overnight at $4^{\circ} \mathrm{C}$. The samples were then resuspended in $0.5 \mathrm{~mL}$ of SM buffer and the concentrated phage solution was stored at $4^{\circ} \mathrm{C}$.

\section{Phage Host Range Test}

A host range test was performed with SFPH2 on 131 S. flexneri, 20 Escherichia coli, 12 Salmonella typhimurium, one Salmonella enteritidis, and three Citrobacter freundii strains isolated from the fecal samples of patients with diarrhea or dysentery. The sensitivity of the clinical strains to the phage was tested by the double-layer agar plate method, which was repeated three times. All the bacterial strains to be tested were grown overnight (14 h) at $37^{\circ} \mathrm{C}$ and $200 \mu \mathrm{l}$ of each of those cultures was used in double layer plaque assays together with $100 \mu \mathrm{l}$ of diluted phage lysate. The phage lysates were diluted from the phage stock and the titer ranged from $10^{2}$ to $10^{4} \mathrm{PFU} / \mathrm{ml}$. In order to investigate the ability of SFPH2 to produce progeny in different strains, the efficiency of plating $(\mathrm{EOP}=$ phage titer on target bacteria/phage titer on host 
bacteria) was calculated as previously described (Khan Mirzaei and Nilsson, 2015).

\section{Transmission Electron Microscopy}

Purified phages (approx. $10^{10} \mathrm{PFU} / \mathrm{mL}$ ) were applied to a carbon-coated copper grid and stained with $2 \%(\mathrm{w} / \mathrm{v})$ uranyl acetate for $40 \mathrm{~s}$. Prepared phage samples were viewed using a transmission electron microscope (JEOL JEM-1200EX, Tokyo, Japan) at $100 \mathrm{kV}$.

\section{Thermal and pH Stability Tests}

For thermal stability assessment, the phage preparation $\left(1 \times 10^{10}\right.$ $\mathrm{PFU} / \mathrm{mL}$ ) was incubated at 37,50 , or $70^{\circ} \mathrm{C}$ for $24 \mathrm{~h}$. For $\mathrm{pH}$ stability studies, phage at $1 \times 10^{10} \mathrm{PFU} / \mathrm{mL}$ was incubated at $\mathrm{pH}$ 2-13 for $24 \mathrm{~h}$. For all experiments, samples were taken at the 1, $3,6,9$, and $24 \mathrm{~h}$ time-points and the phage titer was determined using the double-agar-layer method.

\section{One-Step Growth Curve}

To determine the optimal MOI, serial dilutions of S. flexneri strain SF2 in exponential growth phase were added to aliquots of the SFPH2 stock solution so that the ratio of bacteriophage to host strain was 100,10,1,0.1, and 0.01, respectively. After $5 \mathrm{~min}$ of adsorption, free bacteriophages were removed by centrifugation at $12,000 \times \mathrm{g}$ for $30 \mathrm{~s}$, pellets were resuspended in LB medium, and samples were analyzed for bacteriophage titer using the double-layer agar technique after seven hours of incubation at $37^{\circ} \mathrm{C}$. The solution with a ratio of bacteriophage to host strain that had the highest bacteriophage titer was chosen as having the optimal MOI. To obtain the one-step growth curve, the phage was mixed with the host strain in the exponential phase at the optimal MOI and incubated at $37^{\circ} \mathrm{C}$ for $5 \mathrm{~min}$, then centrifuged at $12,000 \times g$ for $30 \mathrm{~s}$ to remove unabsorbed free phage. The precipitate was washed with LB broth $\left(37^{\circ} \mathrm{C}\right)$ and then transferred into $20 \mathrm{~mL}$ of LB broth followed by incubation at $37^{\circ} \mathrm{C}$. This time-point was defined as $t=0 \mathrm{~s}$ and every $10 \mathrm{~min}$ thereafter, a $0.5 \mathrm{~mL}$ sample was collected for a total duration of $100 \mathrm{~min}$. The titration of phage particles was conducted using the double-layer agar method. The experiment was repeated three times.

\section{Phage DNA Preparation and Sequencing Analysis}

The genomic DNA of phage SFPH2 was extracted following the method described by Wilcox et al. (Wilcox et al., 1996); the genome was sequenced using an Illumina Genome analyzer (Illumina, Inc., San Diego, CA, United States) with approximately 245 -fold coverage. Gap filling was carried out using standard PCR and subsequent Sanger sequencing. The whole-genome sequence was analyzed using the CLC Genomics Workbench 9.0.1 (CLC Bio, Qiagen Bioinformatics, Germany). ORFs were predicted using the NCBI ORF Finder (NCBI Resource Coordinators, 2018) and the complete phage sequence was annotated using the online annotation server RAST (Aziz et al., 2008). The tRNAscan-SE software was used to search for putative tRNA genes (Lowe and Eddy, 1997). A map of the complete SFPH2 genome was constructed using SnapGene software 3.2.1 (GSL Biotech, United States). Sequence comparisons and sequence map generation were performed using BLAST ${ }^{1}$ and Easyfig (Sullivan et al., 2011), respectively. Sequence comparison of tail fiber proteins was performed using the online PredictProtein Tool (Yachdav et al., 2014).

\section{Nucleotide Sequence Accession Number}

The complete genome sequence of SFPH2 is accessible at the GenBank database under accession number MH464253.

\section{RESULTS}

\section{Bacterial Strains}

A total of 131 S. flexneri strains belonging to 15 serotypes were isolated from the fecal samples of patients with diarrhea or dysentery (Table 1). Of these, one serotype 2a strain, SF2, was identified as a multidrug-resistant bacterium, with resistance to aminoglycosides, amphenicols, penicillins, quinolones, and tetracyclines. This SF2 strain was used to isolate the bacteriophage, SFPH2. The remaining 130 strains were used to determine the host range.

\section{Morphology and Host Range Test}

Phage SFPH2 formed clear plaques, $\sim 2 \mathrm{~mm}$ in diameter, on its host bacterial strain on a double-layered agar plate following 6-h incubation at $37^{\circ} \mathrm{C}$. Transmission electron microscopy of the purified and concentrated phage particles showed that phage SFPH2 has an icosahedral capsid, $\sim 50 \mathrm{~nm}$ in diameter, and a short non-contractile tail (Figure 1), matching the typical morphological features of the Podoviridae virus family.

${ }^{1}$ http://blast.ncbi.nlm.nih.gov

TABLE 1 | Host range of Shigella phage SFPH2.

\begin{tabular}{|c|c|c|c|}
\hline S. fexneri serotypes & Spectrum* & EOP & Notes + \\
\hline $1 a$ & $0 / 6$ & - & - \\
\hline $1 b$ & $0 / 5$ & - & - \\
\hline $1 c$ & $0 / 10$ & - & - \\
\hline $2 a$ & $25 / 25$ & $0.36-1$ & Medium/High production \\
\hline $2 \mathrm{~b}$ & $0 / 6$ & - & - \\
\hline 2 variant & $8 / 10$ & $0.14-0.25$ & Medium production \\
\hline $3 a$ & $0 / 6$ & - & - \\
\hline $3 b$ & $0 / 4$ & - & - \\
\hline $4 a$ & $0 / 10$ & - & - \\
\hline $4 a v$ & $0 / 15$ & - & - \\
\hline $4 b$ & $0 / 6$ & - & - \\
\hline$x$ & $0 / 10$ & - & - \\
\hline$X v$ & $0 / 6$ & - & - \\
\hline Y & $3 / 8$ & $0.16-0.23$ & Medium production \\
\hline F6 & $0 / 4$ & - & - \\
\hline
\end{tabular}

"**" The numerator represents the number of strains that can be infected by SFPH2. The denominator represents the total number of strains used in this test. " + " $E O P>0.5$ : High production; $0.1<E O P<0.5$ : Medium production Niazis et al., 2011). The EOP values on each strain are listed in Supplementary Table S1. 


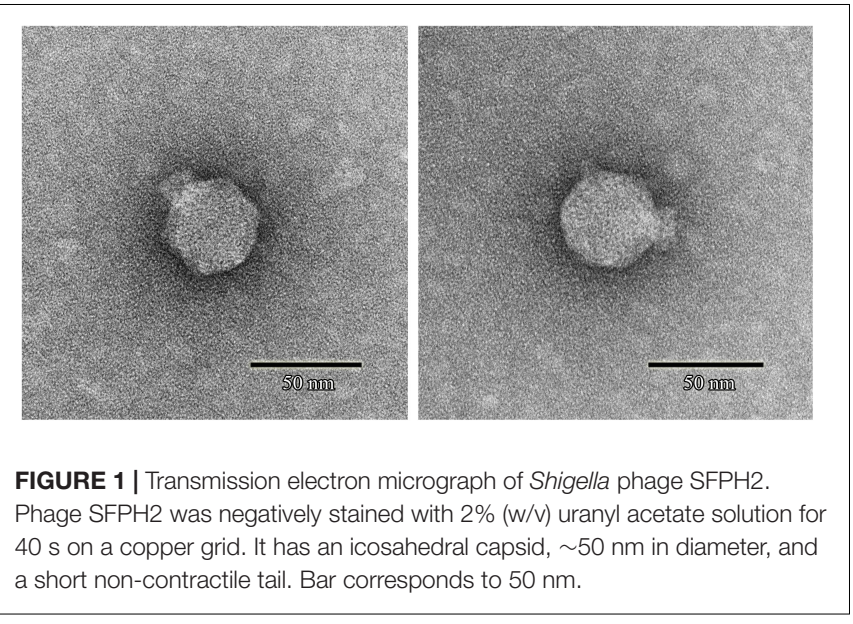

A total of $131 S$. flexneri strains belonging to 15 serotypes were used to determine the host range of SFPH2. All $25 \mathrm{~S}$. flexneri serotype $2 \mathrm{a}$ strains and a number of serotype 2 variant and $\mathrm{Y}$ strains could be lysed by SFPH2, suggesting that this phage has a broad host range in $S$. flexneri 2a strains and limited host range in serotype 2 variant and Y strains (Table 1). Results of the EOP value of SFPH2 on these strains indicated that the phage could cause "medium / high production" efficiency on S. flexneri serotype $2 \mathrm{a}$ strains and "medium production" efficiency on serotype 2 variant and $\mathrm{Y}$ strains according to the classification rules of EOP (Viazis et al., 2011; Table 1 and Supplementary Table S1). In order to test whether SFPH2 can infect other species of bacteria, 20 Escherichia coli, 12 Salmonella typhimurium, one Salmonella enteritidis, and three Citrobacter freundii strains were also used to perform the host range test. However, SFPH2 could not lyse any of these strains (data not shown).

\section{Host Cell Lytic Activity Test}

The multiplicity of infection (MOI) test showed that an MOI of 0.1 yielded the highest level of phage progeny production, $1.62 \times 10^{10}$ plaque forming units (PFU)/mL (Supplementary Table S2). This optimal MOI of SFPH2 was used to generate a one-step growth curve, which was conducted with its host strain SF2 and showed that SFPH2 has a 30-min latent period and a 40-min outbreak period (Figure 2). The maximum number of progeny released from one host bacterium was calculated as the phage burst titer $\left(1.86 \times 10^{10} \mathrm{PFU} / \mathrm{mL}\right)$ divided by the bacterial infection titer $\left(6.23 \times 10^{8}\right.$ colony forming units $\left.(\mathrm{CFU}) / \mathrm{mL}\right)$ and was estimated to be $30 \mathrm{PFU} / \mathrm{cell}$.

\section{Thermal and pH Stability Test}

To investigate the stability of SFPH2 under various environmental conditions, we analyzed its stability at different temperatures and $\mathrm{pH}$ values. SFPH2 retained high activity following incubation at $37^{\circ} \mathrm{C}$ and nearly $80 \%$ of the phages were viable after $24 \mathrm{~h}$ incubation at $50^{\circ} \mathrm{C}$ (Figure 3A). However, no active phages were found after $30 \mathrm{~min}$ at $70^{\circ} \mathrm{C}$. In the $\mathrm{pH}$ stability test, SFPH2 phage remained unaffected over a $\mathrm{pH}$ range of 3-11 after $24 \mathrm{~h}$, exhibiting high stability within this $\mathrm{pH}$ range. At $\mathrm{pH}$

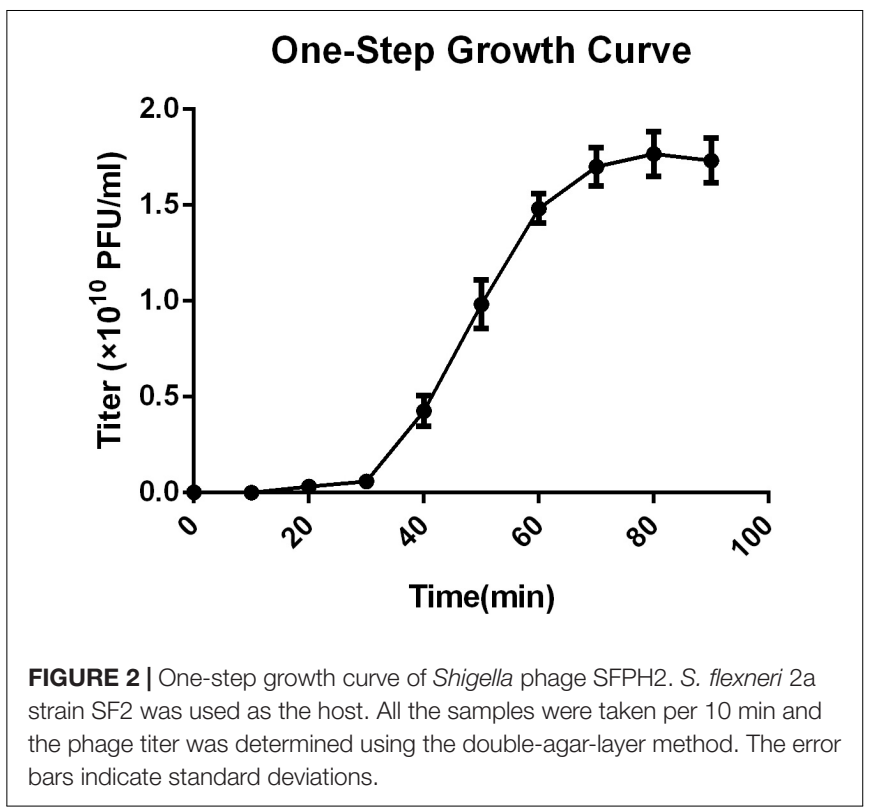

A Thermal Stability Test

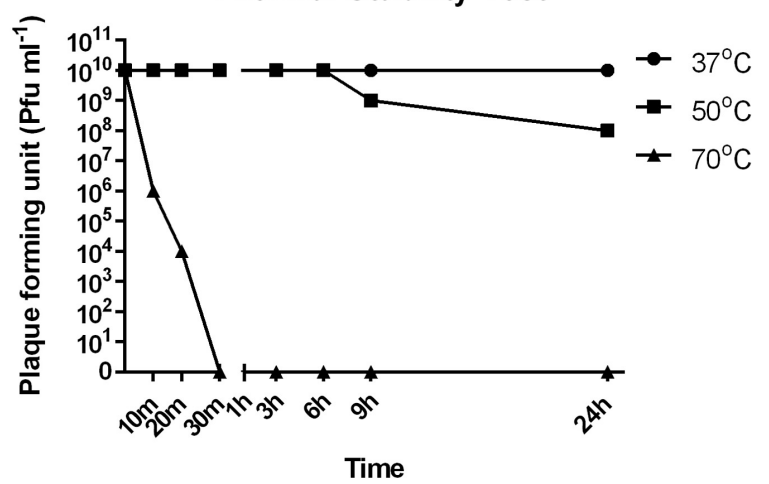

B

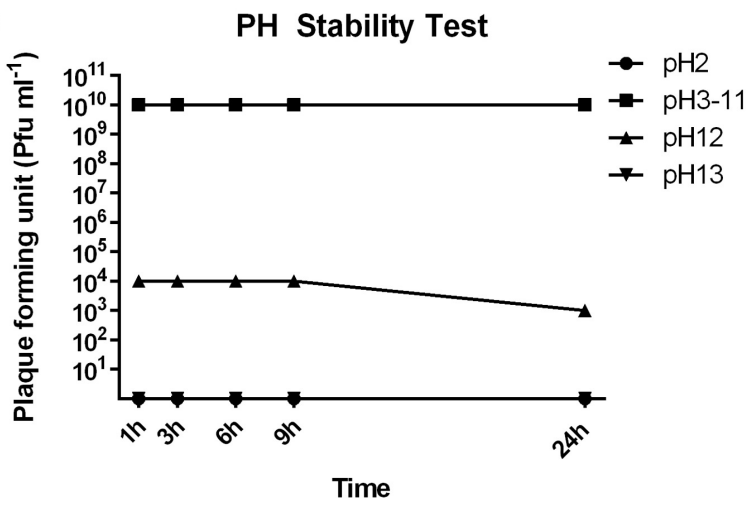

FIGURE 3 | Thermal and pH stability of Shigella phage SFPH2. S. flexneri 2a strain SF2 was used as the host. (A) Thermal stability test. Phage SFPH2 preparation $\left(1 \times 10^{10} \mathrm{Pfu} / \mathrm{ml}\right)$ was incubated at 37,50 , or $70^{\circ} \mathrm{C}$ for $24 \mathrm{~h}$. (B) PH stability test. Phage SFPH2 at $1 \times 10^{10} \mathrm{Pfu} / \mathrm{ml}$ was incubated at $\mathrm{pH}$ 2-13 for $24 \mathrm{~h}$. All the samples of these two tests were taken at 1, 3, 6, 9, and $24 \mathrm{~h}$ time-points and the phage titer was determined using the double-agar-layer method. 
$12,30-40 \%$ of the phages were still viable. However, it showed 0 titer at $\mathrm{pH} 2$ and 13 (Figure 3B).

\section{Characterization of the Phage SFPH2 Genome}

Phage SFPH2 has a linear double-stranded DNA genome, 40,387 bp in length with a $\mathrm{G}+\mathrm{C}$ composition of $52.4 \%$. Of the 50 total putative open reading frames (ORFs), nine (18\%) denote hypothetical proteins and 14 (28\%) were designated as phage proteins with no specific functional annotations. Twentyseven ORFs likely encode functional proteins. These ORFs were divided into three putative functional protein groups: (i) lyticrelated proteins (such as gp38, Phage lysin, N-acetylmuramoylL-alanine amidase), (ii) DNA replication and metabolism-related proteins (such as gp29, phage exonuclease; gp34, T7-like phage
DNA polymerase; gp36, T7-like phage primase/helicase protein; gp40, T7-like phage endonuclease; gp45, DNA ligase), and (iii) morphogenesis-related proteins (such as gp14, phage tail fibers; gp19 and gp20, T7-like tail tubular; gp22 and gp23, phage capsid and scaffold; gp24, phage collar/T7-like phage head-to-tail joining protein) (Figure 4). No tRNA genes were found in the genome.

A similarity comparison with phage proteins in the NCBI database showed that the SFPH2 genome belongs to the subfamily Autographivirinae in the family Podoviridae, with the highest similarity to the T7 branch. The highest similarity throughout was observed with the enterobacterial T7virus, Citrobacter phage SH4 (95\% identity and 89\% coverage, GenBank accession no. KU687350) and Cronobacter phage Dev2 (94\% identity and 92\% coverage, GenBank accession no. HG813241). The genome size (approx. 39-40 kb) of SFPH2 is very similar to

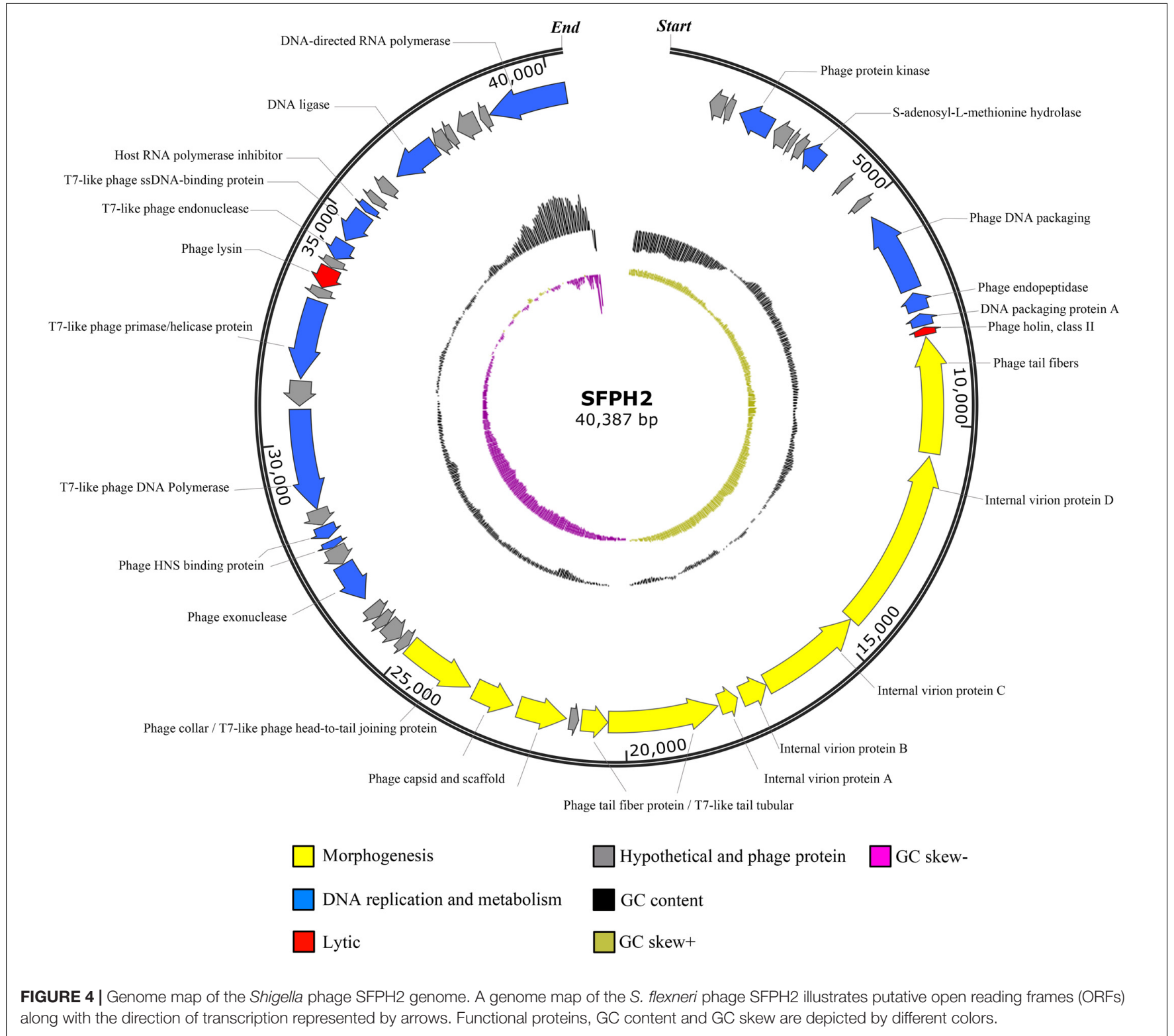




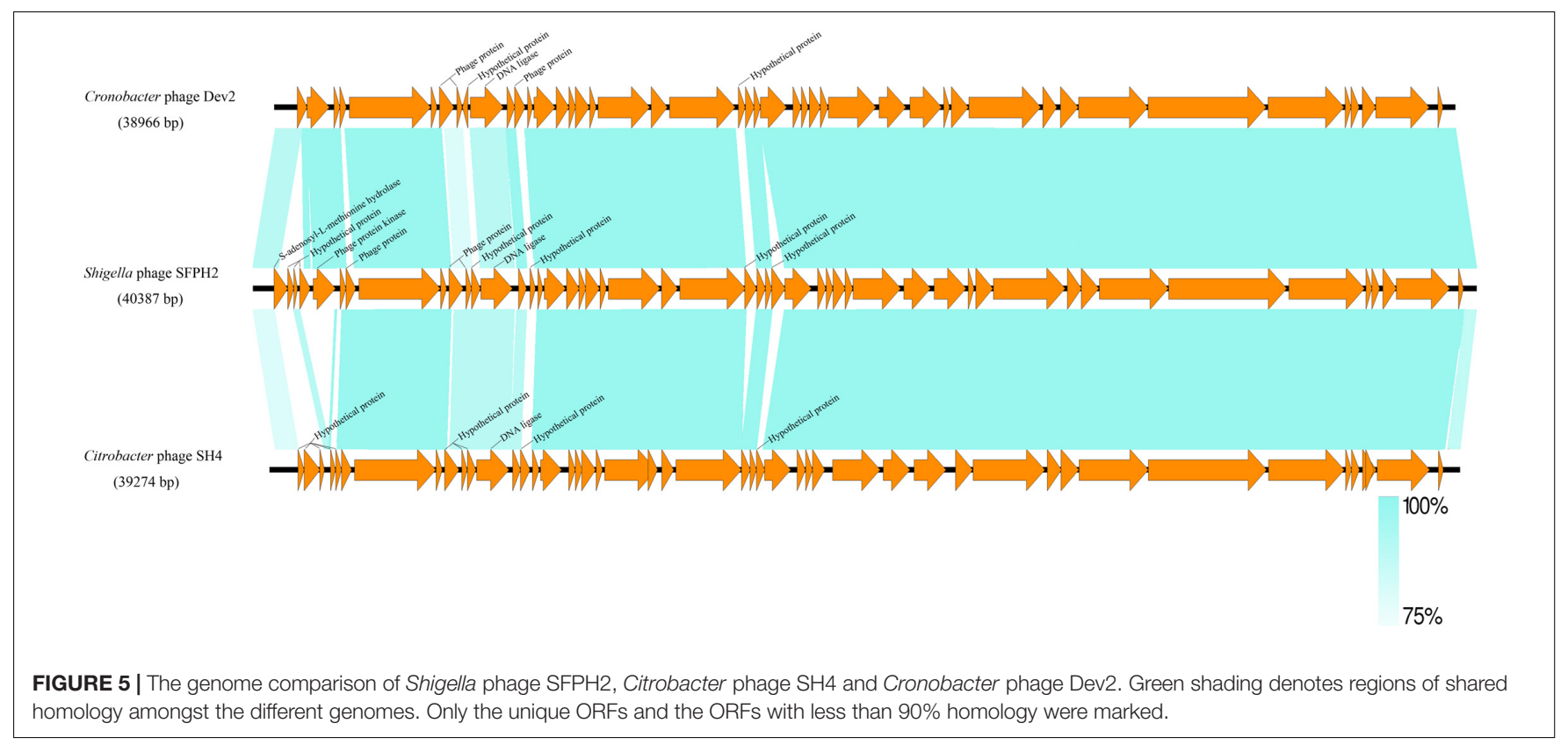

Shigella phage SFPH2
Citrobacter phage SH4
Cronobacter phage Dev2
FIGURE 6| The predicted features comparison of fiber proteins of Shigella phage SFPH2, Citrobacter phage SH4 and Cronobacter phage Dev2. The red diamond
represents protein-binding region; the red and blue rectangle represents the helix and strand of protein, respectively.

that of the two T7virus genomes. SFPH2 was shown to have a linear genome by restriction enzyme digestion (data not shown), and the other two phages also have a linear genome. The genome comparison of these three phages showed that one encoded functional protein, viz. S-adenosyl-L-methionine hydrolase, and several hypothetical phage proteins are unique to SFPH2. The remaining ORFs are homologous or have high similarity to the Citrobacter phage SH4 and Cronobacter phage Dev2 genomes (Figure 5).

Moreover, as tail fiber proteins have been associated with host range determination, the sequences and predicted features of the tail fiber proteins of these three phages were also compared. The results showed that the tail fiber proteins have high similarities
(SFPH2: SH4 97\% similarity; SFPH2: Dev2 96\% similarity) and that the position of the helix and strand in the protein are also substantially similar. However, 27 and 33 amino acid residues of the SFPH2 tail fiber protein sequence differ to those in phages SH4 and Dev2, which might result in differences in their protein binding regions (Figure 6 and Supplementary Figure S1).

\section{DISCUSSION}

Antimicrobial resistance (AMR) has been recognized as a significant global threat to human health (WHO, 2018). Moreover, the rate of multidrug resistance $(\mathrm{MDR}$, resistance to 


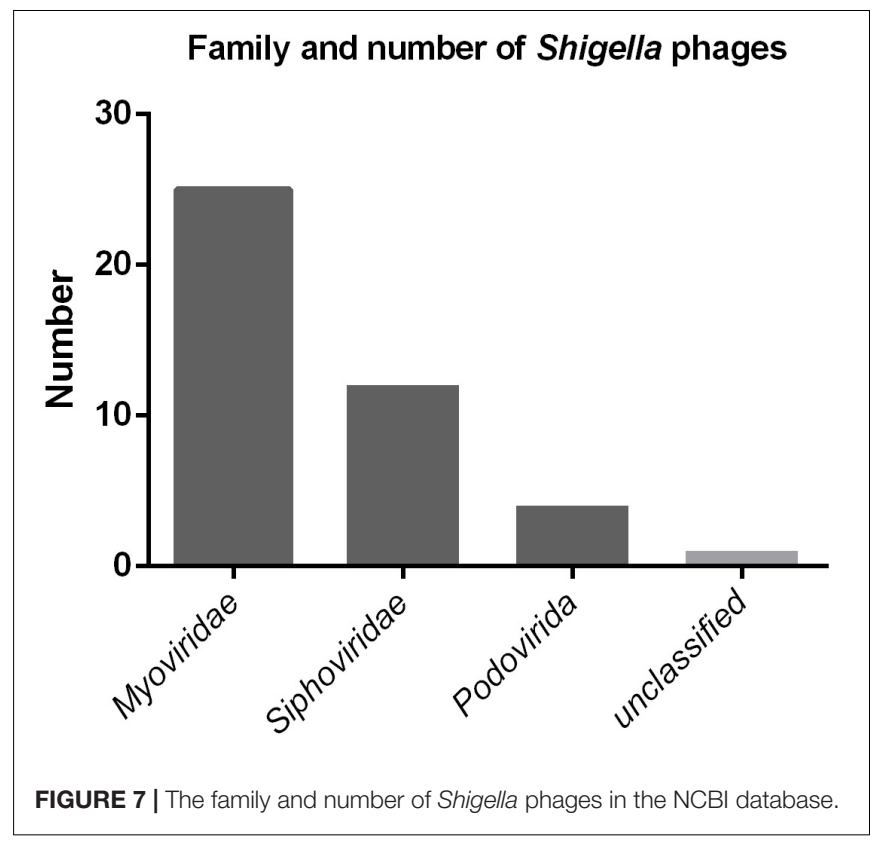

three or more classes of antimicrobials) in Enterobacteriaceae, such as Shigella, is increasing alarmingly because of the widespread and indiscriminate use of antibiotics (Zhang et al., 2011; Nuesch-Inderbinen et al., 2016). In this study, we collected and analyzed 131 S. flexneri strains, 95\% of which were found to be multidrug-resistant (data not shown). Phages have been proposed as alternative agents to protect against and treat infectious diseases caused by AMR pathogens (Sulakvelidze et al., 2001). The aim of this study was to isolate and characterize phage(s) that target multidrug-resistant $S$. flexneri and evaluate their effectiveness as antimicrobial agents to combat these bacteria.

To date, a total of 42 Shigella phages are available in the NCBI database (Supplementary Table S3). The sizes of their genome range between $39.8 \mathrm{kbp}$ and $170.7 \mathrm{kbp}$. According to the classification standards of the ICTV (International Committee on Taxonomy of Viruses) (ICTV, 2017), they all belong to the order Caudovirales. At the family level, there are 25, 12, and five Shigella phages belonging to the family Myoviridae, Siphoviridae, and Podoviridae, respectively (Figure 7). Of the five family Podoviridae phages, three belong to the subfamily Sepvirinae and one belongs to the subfamily Enquartavirinae (Gray et al., 2014; Jun et al., 2014). SFPH2 identified in this study is the only phage known to belong to the subfamily Autographivirinae and genus T7virus (Table 2). The genus T7virus was first accepted under the name $T 7$ phage group and not assigned to any family according to the ICTV Second Report in 1976. The genus was classified into the family Podoviridae in 1981 and renamed twice as T7-like phages and T7likevirus by the ICTV in 1995 and 1999, respectively. The latest renaming of this genus, T7virus, was conducted in 2015. Although 42 years have passed since its first designation, only 88 complete T7virus genomes have been analyzed and submitted to the NCBI database (NCBI Resource Coordinators, 2018), including the genomes of Citrobacter, Pseudomonas, Salmonella and Escherichia phages. However, to date, no Shigella phage from this genus has been characterized. Given the large number of phages in nature and their powerful potential applications, further discovery and characterization of Shigella phages is essential.

In the therapeutic application of bacteriophages, a broad host range is considered to be more advantageous, equivalent to broad-spectrum antibiotics (Ross et al., 2016). Moreover, the ability to continuously amplify phages themselves at the site of infection is a great advantage of phage therapy (Housby and Mann, 2009). But bacterial killing may induced by high multiplicity virion adsorption and that occurs without phage production, which is called "lysis from without" and happens when an overload of phages simultaneously infects a bacterium (Abedon, 2011). This is obviously less useful for phage therapy. Therefore, determining the infection and replication ability of phages to target strains is very important before their application for therapy. Efficiency of plating (EOP), which is frequently used to identify phages suitable for phage therapy, can reflect the ability of phages to produce progeny in bacteria (Khan Mirzaei and Nilsson, 2015). In this study, we found that SFPH2 was able to infect all serotype $2 \mathrm{a}$ and some serotype 2 variant and Y S. flexneri strains. The EOP value of SFPH2 showed it can keep multiplying within these strains. Therefore, this phage could potentially be used against infections caused by these three serotypes. Moreover, SFPH2 was unable to infect other bacteria, such as E. coli, ensuring that its therapeutic use would not affect non-pathogenic E. coli and likely other natural intestinal flora. A previous study has reported that Shigella transmission is particularly associated with poor sanitation because of the low infectious dose of this pathogen (Esrey et al., 1985). Shigella flexneri strains can survive for several months in contaminated water and foodstuffs (Islam et al., 1993, 1996). The Shigella phage could easily be released into the environment in areas where Shigellosis is endemic. Thus, a powerful tool, such as

TABLE 2 | List of currently known Shigella phages of family Podoviridae.

\begin{tabular}{|c|c|c|c|c|c|c|c|c|}
\hline Phage name & $\begin{array}{c}\text { GenBank } \\
\text { accession no. }\end{array}$ & $\begin{array}{c}\text { Genome } \\
\text { length (bp) }\end{array}$ & $\begin{array}{c}\mathrm{G}+\mathbf{C} \\
\text { content }(\%)\end{array}$ & $\begin{array}{l}\text { No. of } \\
\text { CDSs }\end{array}$ & $\begin{array}{c}\text { Genome } \\
\text { type }\end{array}$ & $\begin{array}{l}\text { Taxonomic } \\
\text { Subfamily }\end{array}$ & $\begin{array}{c}\text { Taxonomic } \\
\text { Genera }\end{array}$ & Reference \\
\hline Shigella phage pSb-1 & KF620435 & 71629 & 42.7 & 103 & circular & Enquartavirinae & G7cvirus & Jun et al., 2014 \\
\hline Shigella phage 75/02 Stx & KF766125 & 60875 & 49.1 & 76 & circular & Sepvirinae & Pocjvirus & Unpublished \\
\hline Shigella phage POCJ13 & KJ603229 & 62699 & 49.3 & 79 & linear & Sepvirinae & Pocjvirus & Gray et al., 2014 \\
\hline Shigella phage Ss-VASD & KR781488 & 62851 & 50.1 & 78 & linear & Sepvirinae & TI2011virus & Unpublished \\
\hline Shigella phage SFPH2 & MH464253 & 40387 & 52.4 & 50 & linear & Autographivirinae & T7virus & This study \\
\hline
\end{tabular}


a bacteriophage, would be extremely useful for controlling the rapid spread of Shigella.

A detailed phage genome analysis provided molecular insight into the differences between SFPH2 and other enterobacterial T7virus phages. The SFPH2 genome has a similar size and high sequence similarity with Citrobacter phage SH4 and Cronobacter phage Dev2. Although the three phage genomes were found to be highly similar, their hosts belong to different species. It has been previously noted that host range is associated with the tail fiber proteins or receptor-binding proteins in some phages (Garcia-Doval and van Raaij, 2013). Sequence divergence in tail fiber proteins will lead to different host specificities. Phages can change or expand their host range by mutation of their tail fiber proteins (Casjens and Molineux, 2012). Our results show that the fiber protein sequences and polypeptide folds of the three T7virus phages are highly similar, except for 3$4 \%$ of the amino acid residues that differ; this may result in different protein binding regions and subsequently determine the range of host species. Seckler et al. also found that although the receptor-binding domains of three P22-like phages have no recognizable amino acid sequence similarity and the host ranges of the phages are different, all three have the same polypeptide fold (Barbirz et al., 2008; Muller et al., 2008). These results suggest that the interaction between the tail fiber protein and the host surface receptor occurs via more specific binding. However, most studies have only examined the conformation of tail fiber proteins and few studies have focused on progression of the binding mechanism between the tail fiber proteins and the receptor on the outer membrane surface of host bacteria. Clearly, mechanisms of host species determination and phage-receptor binding are far from being well understood and will remain fertile research ground for some time to come. Further research on these points will help to develop artificial broad host range phages against multiple bacteria.

\section{REFERENCES}

Abedon, S. T. (2011). Lysis from without. Bacteriophage 1, 46-49. doi: 10.4161/bact. 1.1 .13980

Aziz, R. K., Bartels, D., Best, A. A., DeJongh, M., Disz, T., Edwards, R. A., et al. (2008). The RAST Server: rapid annotations using subsystems technology. BMC Genomics 9:75. doi: 10.1186/1471-2164-9-75

Barbirz, S., Muller, J. J., Uetrecht, C., Clark, A. J., Heinemann, U., and Seckler, R. (2008). Crystal structure of Escherichia coli phage HK620 tailspike: podoviral tailspike endoglycosidase modules are evolutionarily related. Mol. Microbiol. 69, 303-316. doi: 10.1111/j.1365-2958.2008.06311.x

Casjens, S. R., and Molineux, I. J. (2012). Short noncontractile tail machines: adsorption and DNA delivery by podoviruses. Adv. Exp. Med. Biol. 726, $143-$ 179. doi: 10.1007/978-1-4614-0980-9_7

Chibani-Chennoufi, S., Bruttin, A., Dillmann, M. L., and Brussow, H. (2004). Phage-host interaction: an ecological perspective. J. Bacteriol. 186, 3677-3686. doi: 10.1128/jb.186.12.3677-3686.2004

Cui, X., Wang, J., Yang, C., Liang, B., Ma, Q., Yi, S., et al. (2015). Prevalence and antimicrobial resistance of Shigella flexneri serotype 2 variant in China. Front. Microbiol. 6:435. doi: 10.3389/fmicb.2015.00435

d'Herelle, F. (1931). Bacteriophage as a treatment in acute medical and surgical infections. Bull. N. Y. Acad. Med. 7, 329-348.

Esrey, S. A., Feachem, R. G., and Hughes, J. M. (1985). Interventions for the control of diarrhoeal diseases among young children: improving water supplies and excreta disposal facilities. Bull. World Health Organ. 63, 757-772.

\section{CONCLUSION}

In conclusion, we have characterized and sequenced a novel Shigella phage, SFPH2, belonging to the family Podoviridae. We have shown that it lyses $S$. flexneri serotype $2 \mathrm{a}, 2$ variant, and $\mathrm{Y}$ strains and is functionally stable under a wide range of temperatures and $\mathrm{pH}$ conditions. Therefore, it has the potential to be widely applied to control Shigella-associated clinical infections.

\section{AUTHOR CONTRIBUTIONS}

HS and SQ designed the experiments. HW, HM, RB, HL, and BL performed the experiments. LY, JX, and YX analyzed the data. ND and LJ prepared the tables and figures. CY and BL prepared the manuscript. All authors have read and approved the final manuscript.

\section{FUNDING}

This work was supported by the National Key R\&D Program of China (No. 2017YFC1600105), Beijing Municipal Natural Science Foundation (No. 7184257), and the National Nature Science Foundation of China (Nos. 81673237 and 81473023).

\section{SUPPLEMENTARY MATERIAL}

The Supplementary Material for this article can be found online at: https://www.frontiersin.org/articles/10.3389/fmicb. 2018.03027/full\#supplementary-material

Garcia-Doval, C., and van Raaij, M. J. (2013). Bacteriophage receptor recognition and nucleic acid transfer. Subcell. Biochem. 68, 489-518. doi: 10.1007/978-94007-6552-8_17

Gray, M. D., Lampel, K. A., Strockbine, N. A., Fernandez, R. E., MeltonCelsa, A. R., and Maurelli, A. T. (2014). Clinical isolates of Shiga toxin 1a-producing Shigella flexneri with an epidemiological link to recent travel to Hispaniola. Emerg. Infect. Dis. 20, 1669-1677. doi: 10.3201/eid2010. 140292

Housby, J. N., and Mann, N. H. (2009). Phage therapy. Drug Discov. Today 14, 536-540. doi: 10.1016/j.drudis.2009.03.006

Hyman, P., and Abedon, S. T. (2010). Bacteriophage host range and bacterial resistance. Adv. Appl. Microbiol. 70, 217-248. doi: 10.1016/s0065-2164(10) 70007-1

ICTV (2017). International Committee on Taxonomy of Viruses. Virus Taxonomy: 2017 Release. Available at: http://ictvonline.org/virusTaxonomy.asp

Islam, M. S., Hasan, M. K., and Khan, S. I. (1993). Growth and survival of Shigella flexneri in common Bangladeshi foods under various conditions of time and temperature. Appl. Environ. Microbiol. 59, 652-654.

Islam, M. S., Rezwan, F. B., and Khan, S. I. (1996). Survival of Shigella flexneri in artificial aquatic environment: effects of different physicochemical stress factors. J. Diarrhoeal. Dis. Res. 14, 37-40.

Jun, J. W., Yun, S. K., Kim, H. J., Chai, J. Y., and Park, S. C. (2014). Characterization and complete genome sequence of a novel N4-like bacteriophage, pSb-1 infecting Shigella boydii. Res. Microbiol. 165, 671-678. doi: 10.1016/j.resmic. 2014.09.006 
Khan Mirzaei, M., and Nilsson, A. S. (2015). Isolation of phages for phage therapy: a comparison of spot tests and efficiency of plating analyses for determination of host range and efficacy. PLoS One 10:e0118557. doi: 10.1371/journal.pone. 0118557

Kotloff, K. L., Winickoff, J. P., Ivanoff, B., Clemens, J. D., Swerdlow, D. L., Sansonetti, P. J., et al. (1999). Global burden of Shigella infections: implications for vaccine development and implementation of control strategies. Bull. World Health Organ. 77, 651-666.

Kropinski, A. M., Mazzocco, A., Waddell, T. E., Lingohr, E., and Johnson, R. P. (2009). Enumeration of bacteriophages by double agar overlay plaque assay. Methods Mol. Biol. 501, 69-76. doi: 10.1007/978-1-60327-164-6_7

Lowe, T. M., and Eddy, S. R. (1997). tRNAscan-SE: a program for improved detection of transfer RNA genes in genomic sequence. Nucleic Acids Res. 25, 955-964. doi: 10.1093/nar/25.5.955

Muller, J. J., Barbirz, S., Heinle, K., Freiberg, A., Seckler, R., and Heinemann, U. (2008). An intersubunit active site between supercoiled parallel beta helices in the trimeric tailspike endorhamnosidase of Shigella flexneri Phage Sf6. Structure 16, 766-775. doi: 10.1016/j.str.2008.01.019

NCBI (2018). National Center for Biotechnology Information. GenomesGroupT7virus. Available: https://www.ncbi.nlm.nih.gov/genomes/GenomesGroup. cgi? opt=phage\&taxid $=110456$

NCBI Resource Coordinators (2018). Database resources of the national center for biotechnology information. Nucleic Acids Res. 46, D8-D13. doi: 10.1093/nar/ gkx1095

Nuesch-Inderbinen, M., Heini, N., Zurfluh, K., Althaus, D., Hachler, H., and Stephan, R. (2016). Shigella antimicrobial drug resistance mechanisms, 20042014. Emerg. Infect. Dis. 22, 1083-1085. doi: 10.3201/eid2206.152088

Qiu, S., Wang, Y., Xu, X., Li, P., Hao, R., Yang, C., et al. (2013). Multidrug-resistant atypical variants of Shigella flexneri in China. Emerg. Infect. Dis. 19, 1147-1150. doi: 10.3201/eid1907.111221

Qiu, S., Xu, X., Yang, C., Wang, J., Liang, B., Li, P., et al. (2015). Shift in serotype distribution of Shigella species in China, 2003-2013. Clin. Microbiol. Infect. 21, .e255-.e258. doi: 10.1016/j.cmi.2014.10.019

Ross, A., Ward, S., and Hyman, P. (2016). More is better: selecting for broad host range bacteriophages. Front. Microbiol. 7:1352. doi: 10.3389/fmicb.2016. 01352

Sulakvelidze, A., Alavidze, Z., Morris, J. G. Jr. (2001). Bacteriophage therapy. Antimicrob. Agents Chemother. 45, 649-659. doi: 10.1128/aac.45.3.649-659. 2001

Sullivan, M. J., Petty, N. K., and Beatson, S. A. (2011). Easyfig: a genome comparison visualizer. Bioinformatics 27, 1009-1010. doi: 10.1093/ bioinformatics/btr039
Sun, Q., Lan, R., Wang, J., Xia, S., Wang, Y., Wang, Y., et al. (2013). Identification and characterization of a novel Shigella flexneri serotype Yv in China. PLoS One 8:e70238. doi: 10.1371/journal.pone.0070238

Viazis, S., Akhtar, M., Feirtag, J., Brabban, A. D., and Diez-Gonzalez, F. (2011). Isolation and characterization of lytic bacteriophages against enterohaemorrhagic Escherichia coli. J. Appl. Microbiol. 110, 1323-1331. doi: 10.1111/j.1365-2672.2011.04989.x

von Seidlein, L., Kim, D. R., Ali, M., Lee, H., Wang, X., Thiem, V. D., et al. (2006). A multicentre study of Shigella diarrhoea in six Asian countries: disease burden, clinical manifestations, and microbiology. PLoS Med. 3:e30353. doi: 10.1371/journal.pmed.0030353

WHO (2017). Global Prioritylist of Antibiotic-Resistant Bacteria to Guide Research, Discovery, and Development of New Antibiotics [Online]. Available at: http://www.who.int/medicines/publications/WHO-PPL-Short_Summary_ 25Feb-ET_NM_WHO.pdf?ua=1

WHO (2018). World Health Organization. Antimicrobial resistance. Available at: http://www.who.int/mediacentre/factsheets/fs194/en/

Wilcox, S. A., Toder, R., and Foster, J. W. (1996). Rapid isolation of recombinant lambda phage DNA for use in fluorescence in situ hybridization. Chromosome Res. 4, 397-398. doi: 10.1007/BF02257276

Yachdav, G., Kloppmann, E., Kajan, L., Hecht, M., Goldberg, T., Hamp, T., et al. (2014). Predict Protein-an open resource for online prediction of protein structural and functional features. Nucleic Acids Res. 42, W337-W343. doi: 10.1093/nar/gku366

Ye, C., Lan, R., Xia, S., Zhang, J., Sun, Q., Zhang, S., et al. (2010). Emergence of a new multidrug-resistant serotype $\mathrm{X}$ variant in an epidemic clone of Shigella flexneri. J. Clin. Microbiol. 48, 419-426. doi: 10.1128/jcm.00614-09

Zhang, W., Luo, Y., Li, J., Lin, L., Ma, Y., Hu, C., et al. (2011). Wide dissemination of multidrug-resistant Shigella isolates in China. J. Antimicrob. Chemother. 66, 2527-2535. doi: 10.1093/jac/dkr341

Conflict of Interest Statement: The authors declare that the research was conducted in the absence of any commercial or financial relationships that could be construed as a potential conflict of interest.

Copyright (C) 2018 Yang, Wang, Ma, Bao, Liu, Yang, Liang, Jia, Xie, Xiang, Dong, Qiu and Song. This is an open-access article distributed under the terms of the Creative Commons Attribution License (CC BY). The use, distribution or reproduction in other forums is permitted, provided the original author(s) and the copyright owner(s) are credited and that the original publication in this journal is cited, in accordance with accepted academic practice. No use, distribution or reproduction is permitted which does not comply with these terms. 